Post-print of: Catalysis Today volume 180, Issue 1, 17 January 2012, pages 161-166

\title{
Analysis and application of the theories that rationalize the crystalline structures of fluorite- related rare earth oxides
}

C. López-Cartes (a), J.A. Pérez-Omil (b), J.M. Rodríguez-Izquierdo (b), J.J. Calvino (b)

a Departamento de Química Inorgánica, Instituto de Ciencia de Materiales de Sevilla, Centro Mixto Universidad de Sevilla-CSIC, Avda Américo Vespucio 49, 41092 Seville, Spain

b Departamento de Ciencia de Los Materiales e Ingeniería Metalúrgica y Química Inorgánica, Facultad de Ciencias, Universidad de Cádiz, 11510 Puerto Real, Cádiz, Spain

\begin{abstract}
The main current theories dealing with the crystalline structures of the fluorite related rare earth oxides, including those corresponding to reduced oxides, one based on the distribution of the coordination defect inside the fluorite structure, and the other which proposes the establishment of modular sequences constituted by modules with fluorite structure, are presented and comparatively explored in detail. Our in-depth analysis of both approximations indicates that they in fact provide smart and efficient rationalizations of the currently known intermediate rare earth oxides structures. We prove however that the strict application of the principles and rules proposed by each theory does not yield unique and unambiguous results for most of the members of the homologous series, as it has been claimed up to now. Moreover, the controversy about the reliability of these two, apparently different and competing, theories is definitely clarified and the exact equivalence of their structural predictions is demonstrated. Finally, we propose new extra systematization rules, not considered up to now in neither of these theoretical approaches, to overcome the observed limitations to properly rationalize the structure of this so technologically important family of oxides.
\end{abstract}

\section{Keywords}

Oxides with fluorite structure; Lanthanide oxides; Coordination defect; Modular sequence

\section{Introduction}

The oxides of the rare earths in general, and more specifically cerium oxides, either pure or mixed with a transition element or a second $4 f$ element, have been used in a wide variety of applications in the fields of heterogeneous catalysis [1] and [2], fast-ion oxygen conductors [3] or chemical sensors [4]. The interesting functions of these materials stem from the fact that some of these elements ( $\mathrm{Ce}, \mathrm{Tb}$ and $\mathrm{Pr}$ ) have the ability to stabilize both +4 and +3 oxidation state in their oxides.

The lanthanide element-oxygen binary systems, and the diagram phases corresponding to oxygen-cerium, praseodymium or terbium have been well established for compositions 
ranging from the sesquioxides ( $\mathrm{Ln2O3}$ ) to the dioxides ( $\mathrm{LnO2}$ ) [5]. For these systems, within the high temperature region, two disordered areas are found, one called $\sigma$ for oxygen/lanthanide ratios from 1.5 to 1.7, and a second one named as $\alpha$ in the composition ratios from 1.7 to 2.0. At lower temperatures, $\alpha$ region yields a variety of apparently non-stoichiometric phases. The whole set of these phases, with general formulas $\mathrm{LnnO} 2 \mathrm{n}-2 \mathrm{~m}$, is known as the homologous series of rare earth oxides. The first member of this series of reduced lanthanide oxides is iota phase ( $\mathrm{L}$ ) whose formula is Ln7012, and it is followed by compounds with formulae Ln9016 and $\operatorname{Ln} 11020$, zeta $(\zeta)$ and delta $(\delta)$ phases respectively.

Early X-ray studies of some of the members of the series [6] clearly showed the close relationship of these phases to the parent fluorite structure of the fully oxidized phases ( $\mathrm{LnO} 2$ ), but no reasonable results were achieved to determine their structures from single crystal X-ray diffraction data. Fortunately, the application of High Resolution Electron Microscopy and Electron Diffraction techniques has been very suitable to confirm the relationship with the fluorite structure of the dioxides, and to obtain the unit cell parameters of the stable phases that could be prepared [7], [8] and [9]. More recently, the structures of some of the members of the homologous series were further refined by high-resolution neutron powder diffraction [10], [11], [12], [13] and [14].

Given the relevance of redox processes in the catalytic action of these oxides, rationalizing the structure of the members of the homologous series, i.e. improving our knowledge about the basic principles which govern the accommodation of oxygen deficiency in these oxides, seems of utmost importance to understand their macroscopic performance.

A first attempt to rationalize the structural principles of these series of oxides argued that oxygen vacancies could not be present alone in the crystal but, instead, that they should interact and distribute in space in the form of vacancy pairs along the $\left\langle\begin{array}{llll}1 & 1 & 1\end{array}\right\rangle$ direction of the host fluorite structure [6]. According to this idea, all the observed structures would then be based on the so-called Bevan cluster. At high temperature these clusters would be randomly distributed in the structure, but at lower temperature they would order in crystallographic planes parallel to the $\left\langle\begin{array}{llll}1 & 3 & 5\end{array}\right\rangle$ reciprocal direction of the fluorite structure, to generate the members of the homologous series [7]. The refinement of the structures by neutron diffraction studies clearly showed that oxygen vacancies can be accommodated by pairs for the highly reduced phases, but that they were present as single vacancies in the oxygen-rich members of the series. After these results, the different models proposed to rationalize the structures of the reduced phases had to be revisited.

In this paper, two relevant theoretical models - the coordination defect model by Martin and co-workers [15], [16], [17], [18] and [19], and the modular sequence of fluorite structure modules model by Eyring and co-workers [20], [21], [22] and [23] that try to establish some basic criteria to unify the whole set of known structures in the fluorite related oxides are comparatively analyzed and their similarities and limitations to predict the structure of these oxides discussed.

The motivation of this work was, on the one hand, to definitely clarify the on-going controversy set out by the authors of the above mentioned theories about the suitability of their models in solving and predicting the crystalline structures of the intermediate oxides. 
Thus, the major mistakes in the way the Coordination Defect (CDT) and the Modular Sequence (MST) theories have been applied will be discussed in detail. Given that our analysis revealed limitations in the potential of both models to univocally determine the exact arrangement of oxygen vacancies in the structure of both known and unknown members of the homologous series of reduced oxides, we also aimed at finding out additional criteria to complete satisfactorily this task.

\section{Discussion}

\subsection{Coordination defect theory (CDT)}

According to the theory proposed by Martin and co-workers [15], [16], [17], [18] and [19], the vacant position has not independent existence in the fluorite structure. On the contrary, it is strongly coordinated by six near neighboring $\mathrm{O2}$ - anions which form an octahedral structural entity $\square$ O6. This entity which has a significant thermodynamic stability was called coordination defect (CD). A representation of this structural unit is shown in Fig. 1. Taking into account that each small cube that constitutes the $C D$ is an octant of the total fluorite unit cell of $\mathrm{LnO2}$, its composition is given by Ln3.5O6. The CD basic unit is considered in this theory as the building block to obtain the structures of the members included in the homologous series of rare earth element oxides. As depicted in the figure, the octants can present two different orientations with respect to the lanthanide cation sublattice, and therefore the coordination defect could have either $\lambda$ or $\delta$ orientation. Another important aspect of the theory is the topological restrictions that limit the way in which the coordination defects can be distributed inside the fluorite structure to originate the ordered reduced phase. The shortest distance between the centers of two adjacent CDs located at different $\left\{\begin{array}{llll}0 & 0 & 1\end{array}\right\} \mathrm{F}$ octant layers is $1 / 2\left\langle\begin{array}{llll}1 & 1 & 1\end{array}\right\rangle \mathrm{F}$, Fig. $2 \mathrm{~d}$. A close packing of coordination defects in this arrangement yields the structure of iota phase (Ln7012) whose composition is equal to that of the basic unit. In the same octant layer the closest distribution of two CDs corresponds to $1 / 2\left\langle\begin{array}{llll}1 & 2 & 0\end{array}\right\rangle \mathrm{F}$ distance, Fig. $2 a$.

On the basis of this theory, the authors proposed the way in which both known and unknown structures could be obtained [19]. The starting point involves representing a unit cell in two dimensions and using a single layer of octants which is in agreement with the crystallographic data of the known phase and with its chemical composition. The vacancies required to match the stoichiometry of the phase are then added in the form of coordination defects following a series of rules. The resulting structures for iota and zeta phases, the members of the homologous series with $\mathrm{n}$ equal to 7 and 9 respectively, as described in the original paper, are shown in Fig. 3. In this representation, the structures are obtained viewed along the $\left\langle\begin{array}{lll}0 & 0 & 1\end{array}\right)$ F orientation. The labels $(+,-, 2+)$ indicate the height of the different CDs with respect to those located at the corners of the parallelogram in the base octant $\left\{\begin{array}{ll}0 & 0 \\ 1\end{array}\right\} \mathrm{F}$ layer. By applying the rules cited above, only one structure is predicted for the iota phase, which is in good agreement with the refined structure [13]. However, this is not the case for zeta phase. For this last stoichiometry two models emerge from the application of CDT: a so-called model 4 , in which the vacant sites are ordered as strings along $\left\langle\begin{array}{llll}1 & 1 & 1\end{array}\right\rangle \mathrm{F}$ direction, and a model 8/17 which presents discrete pairs of vacant positions.

\subsection{Modular sequence theory (MST)}


The second theory that is discussed in this work was proposed by Eyring and co-workers [20], [21], [22] and [23]. These authors assume that all the structures of the homologous series can be obtained by ordering so-called modules with fluorite structure. Each of these modules, except that named as F module, is deficient in oxygen, they all containing inside not more than two vacancies (see Fig. 4). According to the principles of MST, the generation of oxygen vacancies involves the creation of both charge centers and dipolar moments whose intensities and spatial orientations depend on the nature of the module considered. The ordered distribution of the different types of modules, which in this theory is referred as to modular sequence, will try to compensate these local dipolar moments. Thus, to establish the modular sequence for a particular phase there are some forbidden distributions of adjacent fluorite modules. Examples of allowed and forbidden situations are shown in Fig. 5.

In this theory the authors use the $\left\langle\begin{array}{llll}1 & 1 & 2\end{array}\right\rangle \mathrm{F}$ projection to represent the different structures because this direction is common for the principal c-axes of all the members of the homologous series either with known structures or, at least, phases whose cell parameters have been estimated by electron microscopy studies. Using the cell parameters of a specific phase, and taking into account the equal probability of the eight oxygen positions inside the fluorite unit cell to generate the required vacancies, the modular sequence of a structure with formula LnnO2n-2m can be obtained by ordering a number of $n$ modules along the main fluorite directions. The modular sequences for iota and zeta phases, and their representations along the $\left\langle\begin{array}{lll}1 & 1 & 2\end{array}\right\rangle$ F orientation, are represented in Fig. 6 . These structures were reported by the authors as those resulting from the only possible modular sequence that can be obtained for each of these phases. These proposed structures are in perfect agreement with the refined crystallographic data obtained by neutron powder diffraction [10] and [13].

\subsection{Analysis of $C D$ and $M S$ theories}

In this section a detailed analysis of the way in which the authors of the theories apply the rules they propose to obtain the structures of both known and not refined structures is carried out. As commented before, trying to solve the controversy about the reliability of the exposed theories is the main motivation of this analysis.

The first aspect worth mentioning is the fact that, during the structure build up, the authors of the theory based on the CD did not pay attention to the relative orientation of this vacant entity with respect to the cation sublattice. This idea is illustrated in Fig. 7. The scheme in Fig. 7 a represents the distribution of vacancies for the iota phase, considering $\delta$ orientation for the coordination defect located at the corner of the unit cell. On the other hand, in Fig. 7b, a $\lambda$ type orientation has been used for the vacant sites at the origin of the unit cell. The detailed analysis of both schemes reveals that they correspond to the same model, that corresponding to the refined crystalline structure for iota phase, in which the oxygen vacant positions are interconnected by intermediate metal cation sites in the fluorite structure, leading to pairs of vacancies.

This is not the case for the models proposed for the zeta phase. Two models, labeled as 4 and $8 / 17$ respectively, were found for the Ln9016 stoichiometry by applying the rules proposed in the framework of the CDT. Thus, Fig. 8 shows the result of changing the relative orientation of the coordination defect at the origin of the unit cell. As represented in Fig. 8a and b, the 
application of the CDT to model 4, as it also occurs in the case of iota phase, results only in one structure, with independence of the orientation of CDs with respect to the cation sublattice. The oxygen vacant positions are also in this case connected by an intermediate metal atom of the cation sublattice in both schemes. However, for the model $8 / 17$, the modification of the orientation from $\lambda$ to $\delta$ in the coordination defect at the origin of the cell, yields two different structures, Fig. $8 \mathrm{c}$ and d. The difference between them is the relative position of the pairs of vacancies with respect to the metal cation sublattice. Consequently, not only two models (4 and $8 / 17$ ) can be proposed for zeta phase, but also three different structures could be predicted by correctly applying the principles and rules of the theory.

Similar results are obtained if the analysis is carried out for delta phase, Ln11020. For this less reduced stoichiometry, instead of the three models proposed by the authors, labeled 4, 9/16 and 6/19 respectively [19], five different structural models can be predicted if the rules are correctly applied.

After this analysis, it seems clear that the structural prediction for the members of the homologous series by the coordination defect theory is unique only for the most reduced one, iota phase. The application of the rules to any other of the phases produces more than one possible structure that fully follows the requirements of the theory.

Analogous results are obtained when the principles of the MST are strictly applied to the prediction of the intermediate oxide structures. Apart of the iota phase, that gives a single modular sequence matching quite well the real structure obtained by neutron diffraction technique, the application of this second theory to any other member of the series does not originate a single and unambiguous predicted structure. This fact is clearly contradictory with the idea postulated by the authors in terms of its validity to directly elucidate, without any other consideration, the real structure of any known or unresolved crystalline phase. To illustrate this idea, in Fig. 9 two modular sequences different to that originally proposed in the literature for zeta phase are shown which also fully comply with the set of rules established by MST. These two new possible structures for Ln9016 phase are consistent with the unit cell parameters characteristic of this phase and coherent with the phenomenological principles of the theory. The distributions of the vacant oxygen positions inside the fluorite lattice are in this case different to that observed in the original modular sequence determined for zeta phase by the authors of the theory. Similar conclusions can be obtained from the analysis of the delta phase, in this case being possible to distribute the fluorite structure modules necessary to account for its stoichiometry in five valid and different modular sequences.

\subsection{Comparison of the theories}

In the previous section it has been proved that, with the exception of the iota phase, the application of the set of rules of either the CD or the MS theories does not result for most of the stoichiometries of the reduced intermediate phases in a single structural prediction but, instead, in a set of possibilities which agree with the lattice parameters determined from diffraction data but that differ in the details of the distribution of oxygen vacancies. Moreover, the number of possible structural models increases as the reduction degree of the intermediate oxide decreases, being, for example, three possible structures for zeta phase and five for delta. 
Taking into account that both theories generate the same number of predicted structures, an interesting question to be solved is whether or not they are equivalent. To clarify this crucial point, in Fig. 10 the models coming from the CDT for iota and zeta phases, already presented in Fig. 3 and Fig. 8, have been translated into the modules with fluorite structure used in the MST. Following the columns and rows of this figure, which correspond to the $\left[\begin{array}{lll}1 & 0 & 0\end{array}\right]$ and $\left[\begin{array}{lll}0 & 1 & 0\end{array}\right]$ directions in the fluorite matrix, it is easy to realize that there are straight correspondences between the structural models predicted by both models. Model 4 for zeta phase (Fig. 7a), which is translated in terms of a modular sequence in Fig. 10b, is exactly equivalent to the structure shown in Fig. 9a. Following the same argument, models $8 / 17$ considering $\lambda$ or $\delta$ orientation for de coordination defect located at the origin of the unit cell, are equivalent to the modular sequences shown in Figs. 10a and 6b, respectively.

Consequently it is demonstrated that both theories originate the same number of possible models for a specific phase and there is a one to one correspondence between the models predicted by the two theories. Therefore, both theories, though starting from different basic structural concepts drive to exactly the same structural predictions. Moreover, in neither case a unique and unambiguous prediction can be proposed for most of the intermediate phases.

The key unsolved question that limits the applicability of any of these theories is: what are the additional factors that drive a particular stoichiometry to choose between one of the different possibilities predicted by these theories? The analysis of the predicted structures and the comparison of the distribution of oxygen vacancies with that observed in the Rietveld refined crystalline structures help to put forward reasonable proposals to answer this question.

For that purpose, the distances between the oxygen vacancies for different models have been calculated. Table 1 gathers the results of the calculation carried out on the three predicted models for zeta phase. The models have been labeled in this Table following the nomenclature proposed in the CD approximation. All the data in this table are expressed as vectors in the fluorite cubic structure, so the distances between vacancies are equal to half the modulus of each vector in the table. The analysis of the data contained in the table leads to the idea that in model 8/17 the oxygen vacancies are more spread that in model 4 . In the latter, every vacancy has two others defects at a distance equal to $1 / 2\left\langle\begin{array}{llll}1 & 1 & 1\rangle\end{array}\right\rangle$ F. For $8 / 17$ models only one vacancy is located at such distance.

These results therefore suggest that an overall maximization of distances between vacancies is a key factor which influences the final structural choice. This concept needs however be refined since there is not any difference in the relative distribution of the coordination defects or oxygen vacancies between the $8 / 17(\lambda)$ and $8 / 17(\delta)$ models. The difference between both models is just the relative position of the oxygen vacancies with respect to the metal cation sublattice. In the $8 / 17(\lambda)$ model the pair of vacancies located at $1 / 2\left\langle\begin{array}{lll}1 & 1 & 1\end{array}\right\rangle \mathrm{F}$ distance are not connected by a metal atom. However in the $8 / 17(\delta)$ model both vacancies are separated by a cation of the structure.

When the predicted models are compared with the refined atomic positions for zeta phase [10], it is observed that the $8 / 17(\delta)$ model is the one that reproduces the real structure and it consequently must be considered the one describing the most probable ordering of oxygen 
defects in zeta phase. Similar results are observed when an analogous analysis is carried out for delta phase.

At this point it is possible to establish then some additional rules to complete the equivalent $\mathrm{CD}$ and $\mathrm{MS}$ theories that allow us determining the most probable structure for any member of the homologous series, irrespective that such structure has been refined by diffraction methods or not.

Among the set of predicted models for a specific stoichiometry and crystallographic cell parameters, the most probable structure would correspond to the one that maximizes the distances between adjacent oxygen vacancies. Therefore, for those members whose stoichiometry corresponds to a low reduction degree, the oxygen vacancies will tend to distribute in the structure as single vacancies instead of grouping into pairs.

When distances between vacancies are the shortest possible, $1 / 2\left\langle\begin{array}{lllll}1 & 1 & 1\end{array}\right\rangle \mathrm{F}$, and are consequently distributed by pairs inside the structure, the most likely structure will be that in which these vacancies are interconnected by a metal atom.

\section{Conclusions}

The two theories currently available to rationalize the structures of the reduced intermediate phases of Rare Earth Oxides with fluorite related structures have been analyzed in depth. We have proved that the strict application of the basic rules of both theories, something which surprisingly has been missed in previously reported works, drives to identical structural predictions with independence of the particular theory, CDT or MST, used. Therefore both approaches are fully equivalent and it does not make any sense at all to maintain the controversy about which of them is more precise as structural predictive tool. Moreover, with the only use of the set of rules established by any of these theories, in most cases it is not possible to arrive, starting from a particular stoichiometry, to a unique model which unambiguously pictures the distribution of oxygen vacancies within the fluorite matrix but, instead, to sets of possible structures. The number of candidate structures within these sets increases as the total number of vacancies in the unit cell decreases, i.e. as reduction degree decreases.

Two additional criteria have been added in this work which finally allows us selecting one of the possible structures within these sets as that experimentally expected. With this ultimate addition, any of the theories, MST or CDT, become into successful predictive tools.

In relation to these extra rules, our analysis suggests that both, the exact distribution of distances between oxygen vacancies and the relative position of these vacancies with respect to the metal cation sublattice are key factors to decide on the most adequate spatial pattern to accommodate oxygen deficiency in this family of oxides.

The structure of all the members of the homologous series which have been refined by diffraction methods can be satisfactorily explained, rationalized, on the basis of this expanded version of any of the available theories. Moreover, with this tool we can now not only propose 
models for those members whose structure is currently undetermined but also to use these models as initial guesses, input data, for DFT calculations.

\section{Acknowledgements}

We acknowledge financial support from MICINN-FEDER (Proj. Ref: MAT2008-00889-NAN and CSD2009-00013) and Junta de Andalucia (TEP217 and FQM334). 


\section{References}

[1] A. Trovarelli

Catalysis by Ceria and Related Materials

Imperial College Press, London (2002)

[2] A. Trovarelli, C. De Leitenburg, M. Boaro, G. Dolcetti

Catalysis Today, 50 (1999), p. 353

[3] H. Inaba, H. Tagawa

Solid State Ionics, 83 (1996), p. 1

[4] D. Barreca, E. Comini, A. Gasparotto, C. Maccato, C. Maragno, G. Sberveglieri, E. Tondello Journal of Nanoscience and Nanotechnology, 8 (2008), p. 1012

[5] P. Knappe, L. Eyring

Journal of Solid State Chemistry, 58 (1985), p. 312

[6] D.J.M. Bevan

Journal of Inorganic and Nuclear Chemistry, 1 (1955), p. 49

[7] P. Kunzmann, L. Eyring

Journal of Solid State Chemistry, 14 (1975), p. 229

[8] R.T. Tuenge, L. Eyring

Journal of Solid State Chemistry, 41 (1982), p. 75

[9] E. Schweda, D.J.M. Bevan, L. Eyring

Journal of Solid State Chemistry, 90 (1991), p. 109

[10] J. Zhang, R.B. Von Dreele, L. Eyring

Journal of Solid State Chemistry, 118 (1995), p. 133

[11] J. Zhang, R.B. Von Dreele, L. Eyring

Journal of Solid State Chemistry, 118 (1995), p. 141

[12] J. Zhang, R.B. Von Dreele, L. Eyring

Journal of Solid State Chemistry, 122 (1996), p. 53

[13] J. Zhang, R.B. Von Dreele, L. Eyring 
Journal of Solid State Chemistry, 104 (1993), p. 21

[14] E.A. Kümmerle, G. Heger

Journal of Solid State Chemistry, 147 (1999), p. 485

[15] B.F. Hoskins, R.L. Martin

Journal of the Chemical Society - Dalton Transactions (1975), p. 576

[16] B.F. Hoskins, R.L. Martin

Journal of the Chemical Society - Dalton Transactions (1976), p. 676

[17] B.F. Hoskins, R.L. Martin, D. Taylor

Journal of the Chemical Society - Dalton Transactions (1978), p. 320

[18] R.L. Martin

Journal of the Chemical Society - Dalton Transactions (1974), p. 1335

[19] R.L. Martin

Journal of the Chemical Society - Dalton Transactions (1997), p. 3659

[20] Z.C. Kang, L. Eyring

Australian Journal of Chemistry, 49 (1996), p. 981

[21] Z.C. Kang, L. Eyring

Journal of Alloys and Compounds, 249 (1997), p. 206

[22] Z.C. Kang, L. Eyring

Journal of Alloys and Compounds, 275-277 (1998), p. 30

[23] Z.C. Kang, J. Zhang, L. Eyring

Zeitschrift fur Anorganische und Allgemeine Chemie, 622 (1996), p. 465 


\section{Figure captions}

Figure 1. Schematic representation of the coordination defect (CD) constituted by seven octants of fluorite unit cell and with composition equal to Ln3.506. Both $\lambda$ and $\delta$ orientations are considered for the octants in the fluorite structure.

Figure 2. Representation of relative close distribution of two coordination defects inside the fluorite structure.

Figure 3. Schemes of the representation for iota and zeta phase in the CD theory in which $<0$ 0 1) F projections have been considered.

Figure 4. Representation of the different modulus with fluorite structure used by the MS theory.

Figure 5. Allowed (a) and forbidden (b) adjacent distribution of two modules with fluorite structure.

Figure 6. Modular sequences for iota and zeta phases proposed by the authors of MS theory.

Figure 7. Models for iota phase considering different relative orientation of coordination defect, $\delta$ in (a) and $\lambda$ in (b).

Figure 8. Model 4 for zeta phase considering different relative orientation of coordination defect at the origin of the unit cell, $\delta$ in (a) and $\lambda$ in (b). Model $8 / 17$ for zeta phase considering $\lambda$ relative orientation for $C D(a)$ and $\delta(b)$.

Figure 9. Representation of two new modular sequences for zeta phase.

Figure 10. Translation into modular sequences of $C D$ model for iota phase (a) and model 4 (b) and model $8 / 17$ ( $c$ and d) for zeta phase. 
Table 1. Calculated distances between oxygen vacant positions for predicted structural models for zeta phase. The distances between vacancies are equal to half the modulus of each vector.

\begin{tabular}{|c|c|c|c|c|c|c|c|c|}
\hline \multicolumn{3}{|c|}{ Model 8/17 ( $(\lambda)$} & \multicolumn{3}{|c|}{ Model 8/17 (ס) } & \multicolumn{3}{|c|}{ Model 4} \\
\hline-1 & 1 & 1 & 1 & \begin{tabular}{|l|} 
\\
\end{tabular} & -1 & 11 & -1 & \\
\hline 0 & -2 & 1 & 2 & 0 & 1 & & 1 & \begin{tabular}{|l|}
-1 \\
\end{tabular} \\
\hline 1 & 2 & 0 & 0 & 2 & -1 & 0 & 2 & 1 \\
\hline 2 & -1 & 0 & 0 & -1 & 2 & 0 & -2 & -1 \\
\hline-2 & 0 & -1 & -2 & 1 & 0 & & 1 & 2 \\
\hline 0 & 1 & \begin{tabular}{|l}
-2 \\
\end{tabular} & \begin{tabular}{|l|} 
\\
\end{tabular} & -2 & 0 & 2 & 11 & -1 \\
\hline 1 & 1 & 2 & 1 & 1 & 2 & -1 & $-1]$ & -2 \\
\hline 2 & 1 & -1 & 2 & 1 & -1 & -2 & $-1 \mid$ & 1 \\
\hline-1 & -1 & -2 & -1 & -1 & -2 & \begin{tabular}{|l|}
1 \\
\end{tabular} & 2 & -2 \\
\hline-2 & -1 & 1 & -2 & $\mid-1$ & 1 & 2 & $-1 \mid$ & -2 \\
\hline 1 & -2 & -2 & \begin{tabular}{|l}
-1 \\
\end{tabular} & 2 & 2 & 3 & \begin{tabular}{|l|}
0 \\
\end{tabular} & 0 \\
\hline 1 & 0 & -3 & 1 & 0 & -3 & -1 & \begin{tabular}{|l|}
-2 \\
\end{tabular} & 2 \\
\hline 1 & -3 & 0 & 1 & -3 & 0 & -2 & 1 & 2 \\
\hline-1 & 0 & 3 & -1 & 0 & 3 & & \begin{tabular}{|l|l|}
0 \\
\end{tabular} & 0 \\
\hline-1 & 3 & 0 & -1 & 3 & 0 & 1 & 0 & -3 \\
\hline 1 & -1 & 3 & 1 & 3 & 1 & 1 & $-3 \mid$ & 0 \\
\hline \multirow[t]{2}{*}{-1} & -3 & -1 & -1 & 1 & -3 & -1 & 0 & 3 \\
\hline & & & & & & & 3 & 0 \\
\hline
\end{tabular}


Figure 1

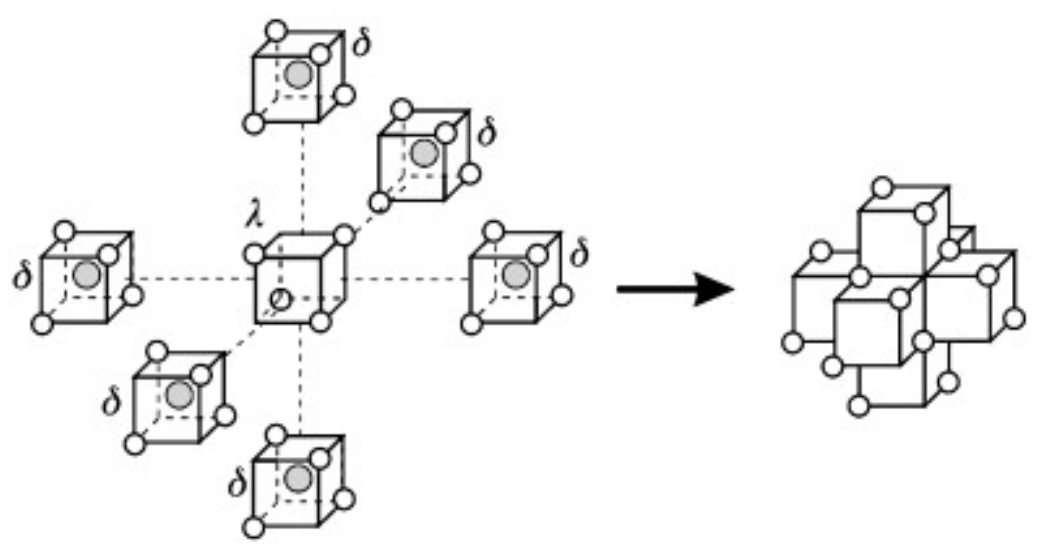


Figure 2
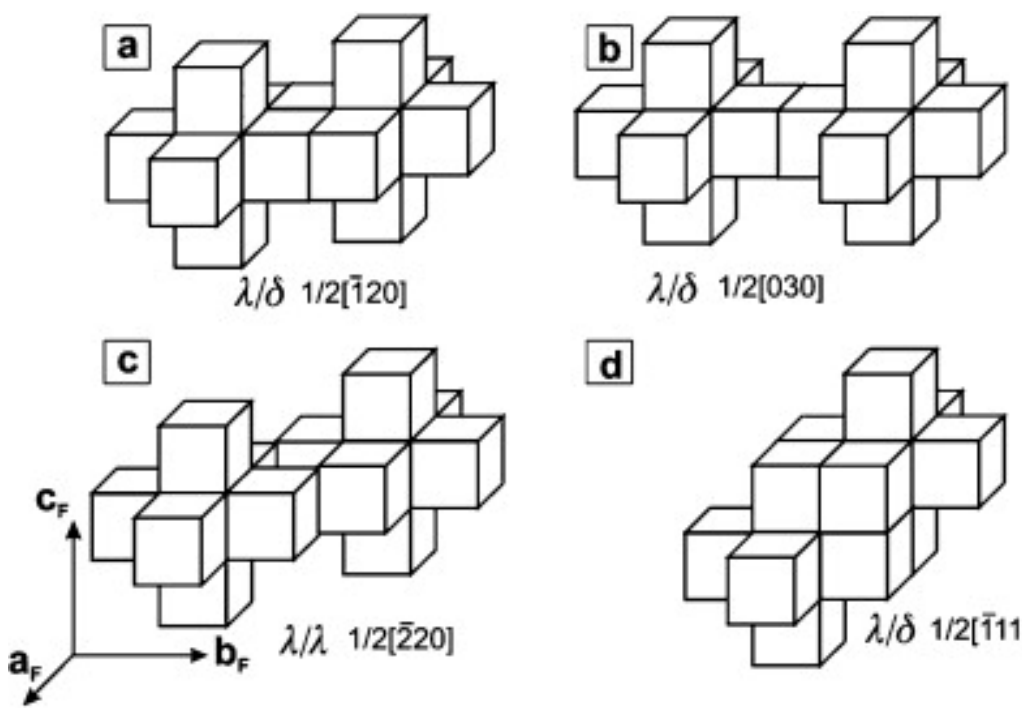

d

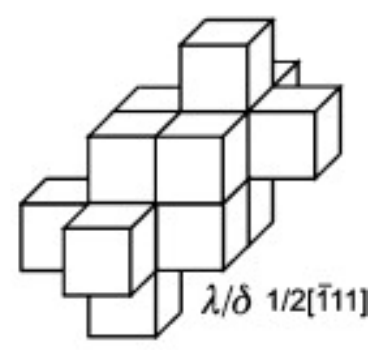


Figure 3

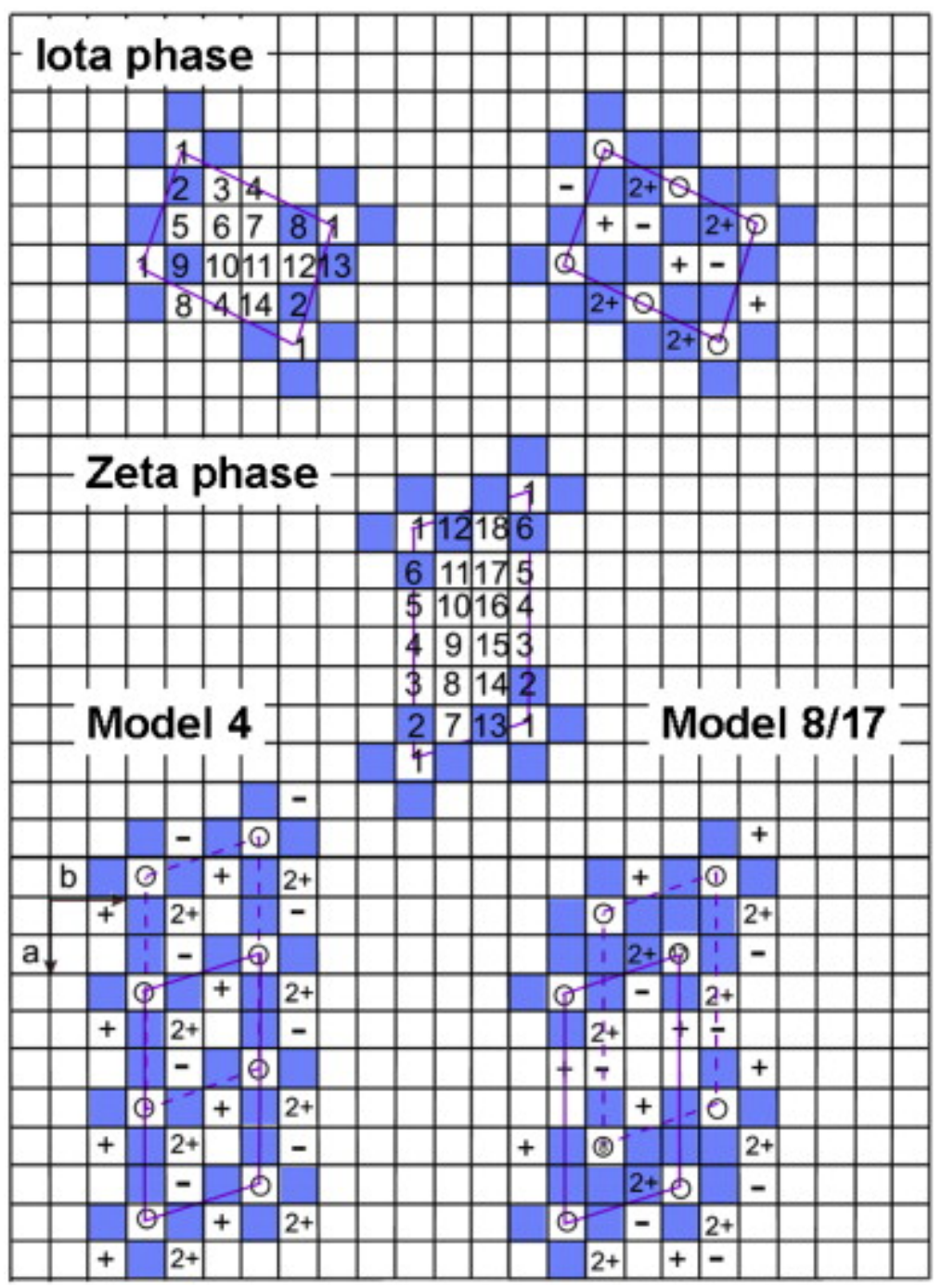


Figure 4
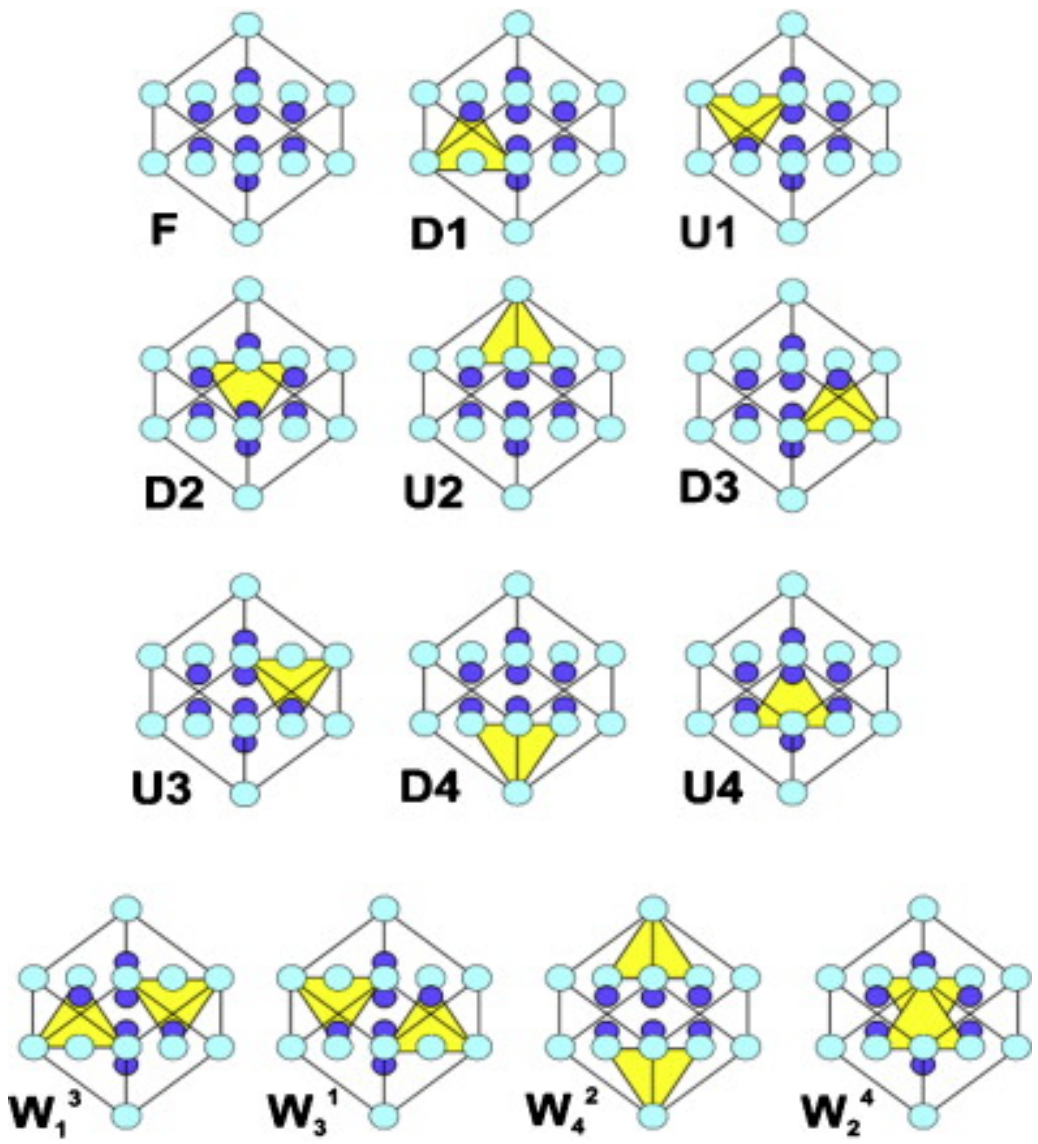
Figure 5
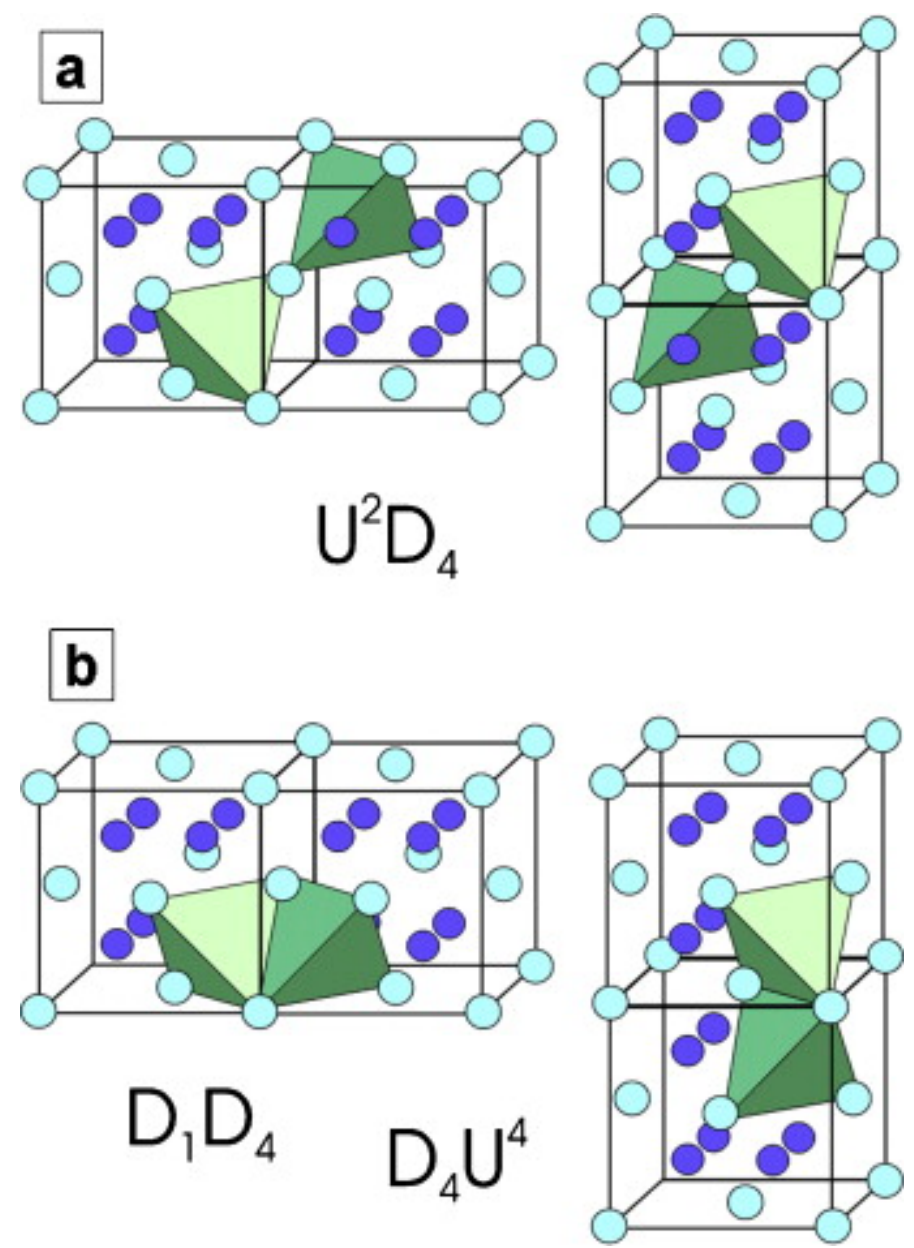
Figure 6

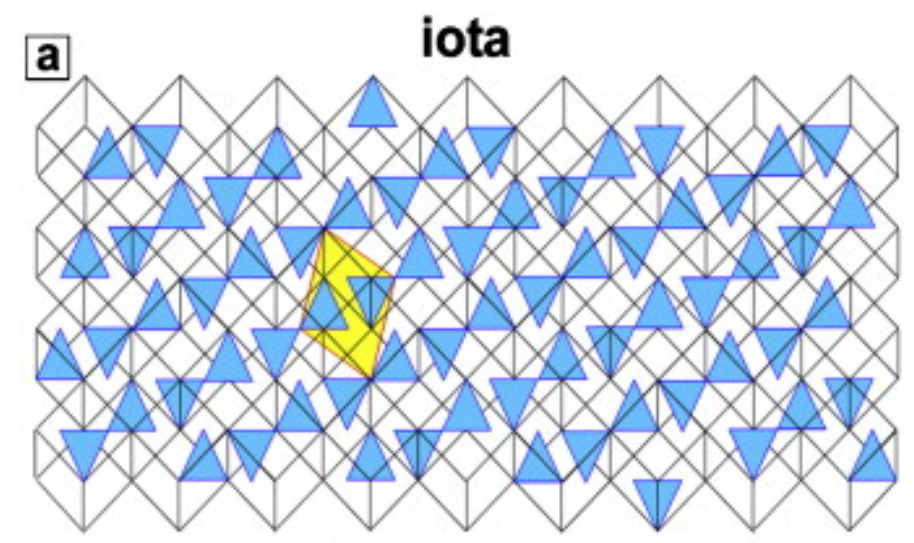

[100] $\mathrm{W}_{1}^{3} \mathrm{U} 1 \mathrm{D} 4 \mathrm{U} 4 \mathrm{D} 2 \mathrm{U} 2 \mathrm{D} 3$

[010] $\mathrm{W}_{1}^{3}$ U4 D3 D4 U2 U1 D2

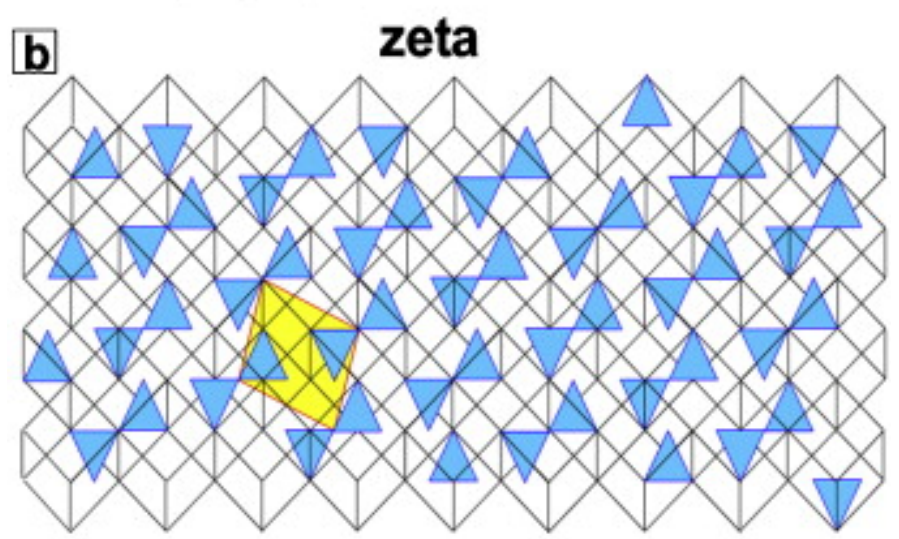

[100] U3 U2 D3 U3 U2 D3 U3 U2 D3

[010] U3 U4 D4 U2 D2 D1 D3 F U1 
Figure 7

$a_{0}^{+0_{0}^{+0}}$


Figure 8

Model 4

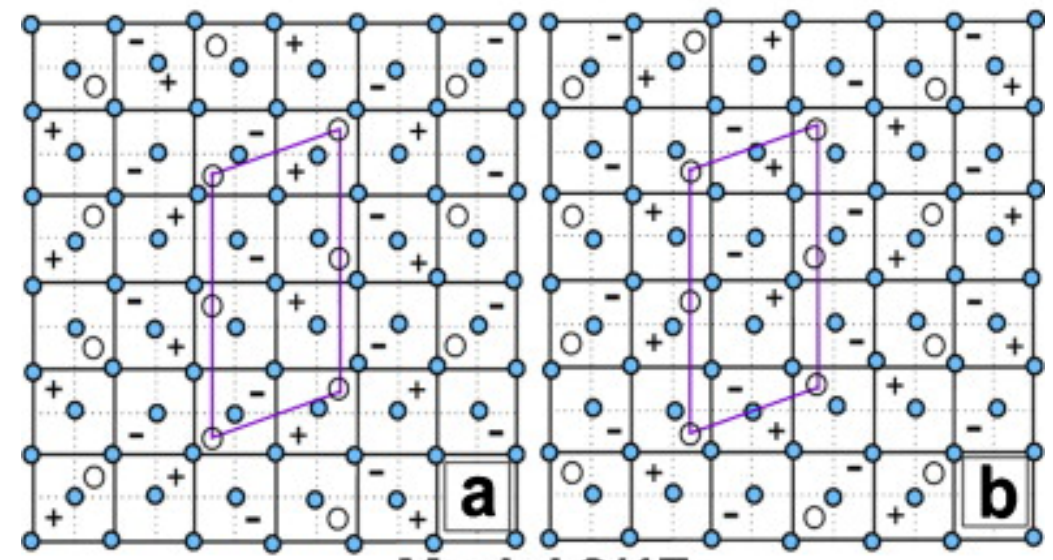

Model 8/17

$\underbrace{+0}_{0}$


Figure 9
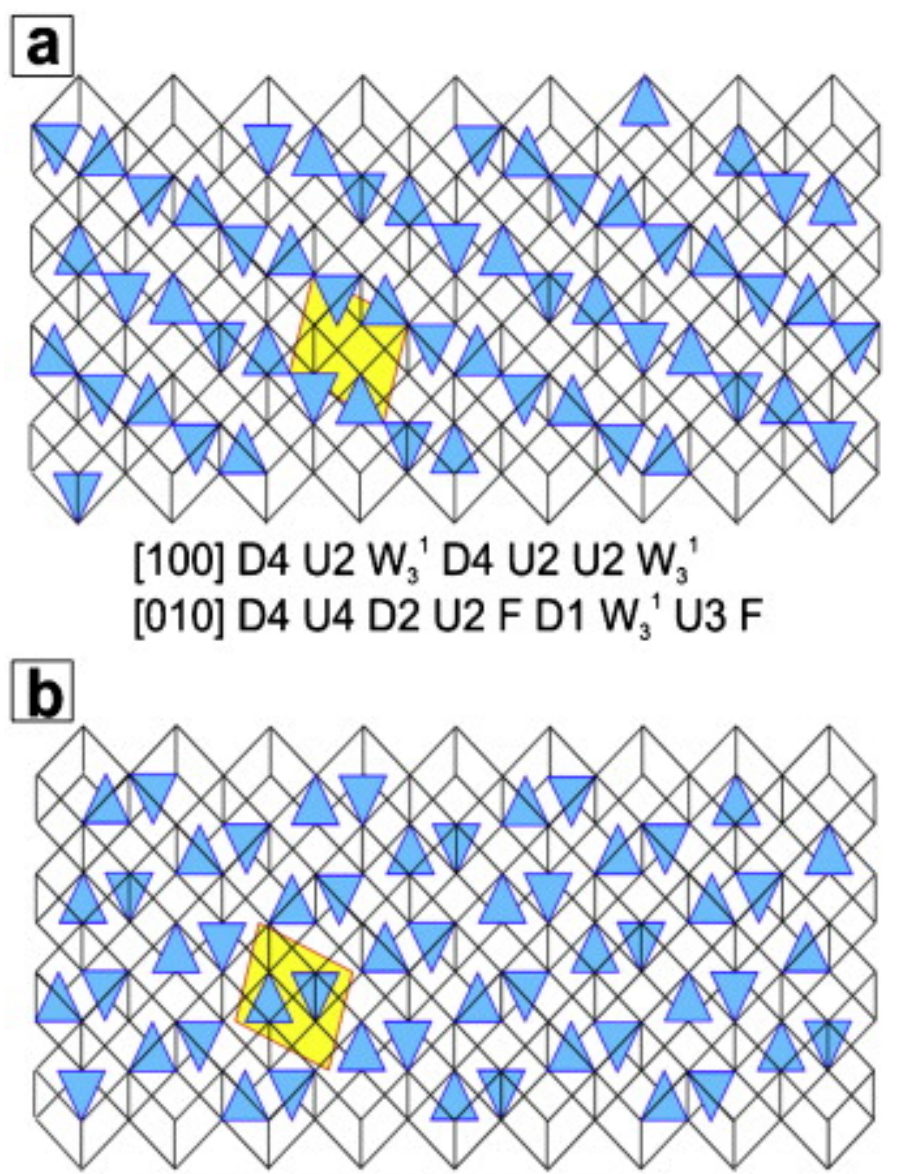

[100] U2 D3 D2 U2 D3 D2

[010] U2 U1 W ${ }_{1}^{3}$ D3 D4 F D2 U4 F 
Figure 10

\begin{tabular}{|c|c|c|c|c|c|c|c|c|c|c|c|}
\hline 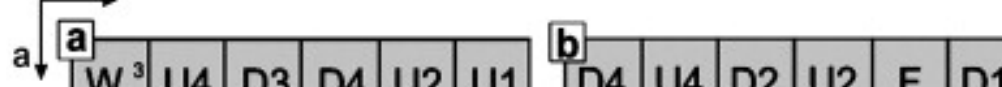 & $\mathrm{U} 4$ & D3 & D4 & U2 & U1 & D4 & U4 & D2 & $\mathrm{U} 2$ & $F$ & D1 \\
\hline U1 & D2 & $W_{1}^{3}$ & U4 & D3 & D4 & U2 & $F$ & D1 & $\mathrm{W}_{3}{ }^{1}$ & U3 & $F$ \\
\hline D4 & $\mathrm{U} 2$ & U1 & D2 & $\mathrm{W}_{1}^{3}$ & U4 & $\mathrm{W}_{3}{ }^{\mathrm{C}}$ & U3 & $\mathrm{F}$ & D4 & U4 & D2 \\
\hline U4 & D3 & D4 & U2 & U1 & D2 & D4 & U4 & D2 & $\mathrm{U} 2$ & $\mathrm{~F}$ & D1 \\
\hline D2 & $W_{1}^{3}$ & U4 & D3 & D4 & $\mathrm{U} 2$ & U2 & $\mathrm{F}$ & D1 & $\mathrm{W}_{3}{ }^{1}$ & U3 & $\mathrm{F}$ \\
\hline U2 & U1 & D2 & $\mathrm{W}_{1}{ }^{3}$ & U4 & D3 & $W_{3}{ }^{\prime}$ & U3 & $\mathrm{F}$ & D4 & U4 & D2 \\
\hline \\
\hline U2 & U1 & $W_{1}^{3}$ & D3 & D4 & $F$ & U3 & U4 & D4 & U2 & D2 & D1 \\
\hline D3 & D4 & $\mathrm{F}$ & D2 & U4 & $F$ & U2 & D2 & D1 & D3 & $F$ & U1 \\
\hline D2 & U4 & $F$ & U2 & $\mathrm{U}_{1}$ & $\mathrm{~W}_{1}^{3}$ & D3 & $\mathrm{F}$ & U1 & U3 & U4 & D4 \\
\hline U2 & U1 & $W_{1}^{3}$ & D3 & D4 & $F$ & U3 & U4 & D4 & $\mathrm{U} 2$ & D2 & D1 \\
\hline D3 & D4 & $\mathrm{F}$ & D2 & U4 & $F$ & U2 & D2 & D1 & D3 & $F$ & U1 \\
\hline D2 & U4 & $F$ & U2 & $\mathrm{U}_{1}$ & $W_{1}^{3}$ & D3 & $F$ & U1 & U3 & U4 & D4 \\
\hline
\end{tabular}


